\title{
Spin chains for two-qubit teleportation
}

\author{
Tony J. G. Apollaro,,${ }^{1}{ }^{*}$ Guilherme M. A. Almeida, ${ }^{2}$ Salvatore Lorenzo, ${ }^{3}$ Alessandro Ferraro, ${ }^{4}$ and Simone Paganelli $\odot^{5}$ \\ ${ }^{1}$ Department of Physics, University of Malta, Msida MSD 2080, Malta \\ ${ }^{2}$ Instituto de Física, Universidade Federal de Alagoas, 57072-900 Maceió, AL, Brazil \\ ${ }^{3}$ Dipartimento di Fisica e Chimica, Universitá degli Studi di Palermo, via Archirafi 36, I-90123 Palermo, Italy \\ ${ }^{4}$ Centre for Theoretical Atomic, Molecular and Optical Physics, Queen's University Belfast, Belfast BT7 1NN, United Kingdom \\ ${ }^{5}$ Dipartimento di Scienze Fisiche e Chimiche, Università dell'Aquila, via Vetoio, I-67010 Coppito-L'Aquila, Italy
}

(Received 26 July 2019; published 6 November 2019)

\begin{abstract}
Generating high-quality multiparticle entanglement between communicating parties is the primary resource in quantum teleportation protocols. To this aim, we show that the natural dynamics of a single spin chain is able to sustain the generation of two pairs of Bell states--possibly shared between a sender and a distant receiver-which can in turn enable two-qubit teleportation. In particular, we address a spin- $\frac{1}{2}$ chain with $X X$ interactions, connecting two pairs of spins located at its boundaries, playing the roles of sender and receiver. In the regime where both end pairs are weakly coupled to the spin chain, it is possible to generate at predefinite times a state that has vanishing infidelity with the product state of two Bell pairs, thereby providing nearly unit fidelity of teleportation. We also derive an effective Hamiltonian via a second-order perturbation approach that faithfully reproduces the dynamics of the full system.
\end{abstract}

DOI: 10.1103/PhysRevA.100.052308

\section{INTRODUCTION}

Quantum information processing (QIP) has become the subject of an increasingly intensive theoretical and experimental effort over the last few decades. With research fields spanning from computation to simulation and metrology, QIP aims at leading the next quantum revolution by developing devices able to outperform any classical analog in a variety of tasks, from cryptographic key distribution to the simulation of chemical reactions. Nevertheless, a necessary condition for almost any QIP task is the capability of implementing a faithful quantum state transfer (QST) protocol [1-3]. Indeed, QIP tasks such as quantum key distribution and quantum computation require the transfer of quantum information from a sender to a receiver, embodied by a measurement apparatus or quantum processors.

The means by which QST is achievable can be grouped in three large classes. The first one involves the physical displacement of a carrier encoding the information (e.g., photons) and has been successfully employed in cavity QEDbased architectures [4,5]. The second one relies on the dynamics of a physical quantum channel connecting the sender and the receiver, the former encoding the information in a stationary qubit at its location, with the aim that the evolution of the quantum channel allows the information to be retrieved at the receiver's stationary qubit location. In this context, spin$\frac{1}{2}$ chains have been intensively investigated as faithful quantum channels for a variety of tasks [6-11]. Finally, the third QST protocol is based on exploiting a preexisting quantum resource, usually entanglement, and perform a teleportation protocol-which represents the most prominent example of

\footnotetext{
*tony.apollaro@um.edu.mt
}

quantum communication under local operations and classical communication (LOCC) constraints. In this paper, we focus on the use of a spin- $\frac{1}{2}$ chain to generate such a quantum resource, which can then be used for the deterministic teleportation of an arbitrary two-qubit state.

Since the seminal work by Bennett et al. that introduced the quantum teleportation protocol of a single qubit via the use of a Bell pair and a classical communication channel [12], great effort has been devoted both to its experimental implementation [13] and to the generalization to higher-dimensional systems - in particular, $n$-qubit teleportation protocols. The latter find a natural application in LOCC-constrained quantum communication, where high-dimensional systems guarantee higher security and increased transmission rates [14-23]. Also, $n$-qubit teleportation protocols can be used in quantum computation, especially in distributed approaches [24] where the state of a quantum register needs to be routed to different processing units-and in client-server models [25] — where quantum computation is performed by a remote unit.

While in the original protocol in Ref. [12] the quantum channel for deterministic teleportation is embodied by one of the Bell states, many other states have been found to achieve the same goal, among which is the three-particle Greenberger-Horne-Zeilinger (GHZ) and a class of $W$ states $[26,27]$. The search for two-qubit teleportation protocols went along the same line: from the original proposal exploiting tensor products of two Bell states $[28,29]$ to genuine four- and five-qubit entangled states [30,31] and a class of four-qubit states having cluster states as a special case [32]. Similarly, for $n$-qubit teleportation, $2 n$-qubit states made up by Bell tensor product states constitute a faithful quantum channel and the necessary and sufficient conditions they have to fulfill are given in Ref. [33]. 
Whereas the generation and distribution of single Bell states-that is, the key resource for one-qubit teleportationhave been widely investigated, the same does not hold for the entangled states needed for $n$-qubit teleportation. In the context of spin chains, several schemes have been proposed to generate a Bell state between two distant qubits [6,8,34-40] based mainly on the same protocol used for one-qubit quantum state transfer. Clearly, any of these schemes could be used to sequentially generate Bell states by removing the entangled spins from the chain and waiting for a new Bell pair to be formed. A drawback of such a procedure is that it requires control over the motional degree of freedom of the spins and the sequential use of a spin chain as a quantum entangler could require its initialization at each run, not to mention that the coherent dynamics of the quantum channel has to be preserved for longer times. It is hence evident that, also due to scalability issues, it would be beneficial to have a single quantum chain able to support the generation of $n$ pairs of Bell states, shared among a sender and a distant receiver, to be used as a resource for the teleportation of $n$ qubits. Recently, the transfer of arbitrary two-qubit states, as well as specific classes thereof, have received a lot of attention [41-48], but the search for a protocol able to generate, via the natural spin chain dynamics, entanglement involving spins at distant locations to serve as a resource for two-qubit teleportation has yet to be addressed.

In the present paper we address such a question. In Refs. [28,29] it was shown that perfect two-qubit teleportation can be achieved by means of a four-qubit maximally entangled state, which is the tensor product of a pair of two-qubit entangled states shared by the sender and receiver blocks. Here, we show how a one-dimensional (1D) spin- $\frac{1}{2}$ chain with nearest-neighbor couplings of the $X X$ type and open boundary conditions can give rise to such a state when the spins residing at the opposite edges of the chain are weakly coupled to the channel. The paper is organized as follows: In Sec. II we introduce the spin model and in Sec. III we work out an effective perturbative Hamiltonian that faithfully reproduces its dynamics; in Sec. IV we evaluate the bipartite entanglement between the two pairs of spins located at the edges of the chain and we show its usefulness for a twoqubit teleportation protocol; finally, in Sec. V we draw our conclusions and outlooks.

\section{THE MODEL}

Our model consists of a 1D spin- $\frac{1}{2}$ chain with open boundaries and an isotropic nearest-neighbor interaction in the $X Y$ directions, where the first two spins are the sender party $\left(A_{1}, A_{2}\right)$, the last two are the receiver party $\left(B_{1}, B_{2}\right)$, and the $M$ spins in between represent the channel,

$$
H=\sum_{i} \frac{J_{i}}{2}\left(\hat{\sigma}_{i}^{x} \hat{\sigma}_{i+1}^{x}+\hat{\sigma}_{i}^{y} \hat{\sigma}_{i+1}^{y}\right),
$$

where $\hat{\sigma}_{i}^{\alpha}(\alpha=x, y, z)$ are the Pauli matrices and index $i$ comprises $A_{1}, A_{2}, 1, \ldots, M, B_{1}$, and $B_{2}$. The Hamiltonian described by Eq. (1) exhibits $U(1)$ symmetry, thus conserving the total magnetization in the $z$ direction, and can be reduced to a model of noninteracting spinless fermions [see Eq. (8)]. We set coupling strengths $J_{i}$ to be uniform along the chain except those interfacing the sender and receiver blocks with the channel, that is, $J_{i}=J$ for $i \neq A_{2}, M$ and $J_{i}=g$ otherwise, with $g \ll J$ and $J=1$ being the energy unit (see Fig. 1). A similar scheme has been found to yield a high-fidelity two-qubit quantum state transfer in Ref. [44].

In our protocol, the initial state of the quantum channel is fully polarized and each pair of qubits located at the edges $A$ and $B$ can be judiciously initialized, that is, $|\Psi\rangle=$ $|\varphi\rangle_{A_{1} A_{2}}|\mathbf{0}\rangle_{1 \ldots M}|\varphi\rangle_{B_{1} B_{2}}$, with $|\mathbf{0}\rangle_{1 \ldots M}=|00 \ldots 0\rangle$ involving all the channel spins. We denote by $|0\rangle$ and $|1\rangle$, respectively, the spin-up $|\uparrow\rangle$ and spin-down $|\downarrow\rangle$ state of a single spin- $\frac{1}{2}$ system. Our primary goal is to achieve a tensor product of two Bell states, which in their generalized version read

$$
\begin{aligned}
& \left|\Phi_{\theta}^{1,2}\right\rangle=\frac{1}{\sqrt{2}}\left(|01\rangle \mp e^{-i \theta}|10\rangle\right), \\
& \left|\Phi_{\theta}^{3,4}\right\rangle=\frac{1}{\sqrt{2}}\left(|00\rangle \mp e^{-i \theta}|11\rangle\right)
\end{aligned}
$$

(note that the entanglement featured in a Bell state is independent of its relative phase) between blocks $A$ and $B$. To do so, we need to determine the Hamiltonian dynamics in the invariant subspaces with $(0,2,4)$, $(1,3)$, or (2) flipped spins, depending on which Bell states enter the product. For instance, if we were considering the tensor product state $\left|\Phi_{\theta}^{1}\right\rangle\left|\Phi_{\phi}^{3}\right\rangle=$ $\frac{1}{2}\left(|0100\rangle-e^{-i \theta}|1000\rangle-e^{-i \phi}|0111\rangle+e^{-i(\theta+\phi)}|1011\rangle\right), \quad$ the dynamics would occur in the Hilbert space with one and three spins down, i.e., in the $(1,3)$-excitation invariant subspaces. Nevertheless, as Bell states are all equivalent under local unitary operations, each of the 16 tensor products can be obtained from an arbitrary one. Clearly, because of the conservation of the total magnetization along the $z$ axis, the initial number of flipped spins is conserved and so in the $A \cup B$ block there have to be $n \geqslant 2$ flipped spins. In the following we investigate the case $n=2$ since, as we show in our perturbation analysis later on, the quantum channel (bulk of the chain) will not effectively support any flipped spin during the dynamics. (Henceforth we omit $|\mathbf{0}\rangle_{1 \ldots M}$ for convenience.) Therefore, in order to possibly generate a tensor product of Bell states lying in subspaces with $(0,2,4)$ or $(1,3)$ flipped spins, the initial state cannot be a simple tensor product of single-qubit states, implying that it should contain some entanglement. Although this could be achieved by exploiting the dynamics of the spins in blocks $A$ and $B$ before coupling them to the quantum channel, it would require additional time control over the couplings if the initial states are not eigenstates of their respective Hamiltonian.

Our analysis is thus made upon initial states of the form (using the notation $\left|s_{A_{1}} s_{A_{2}} s_{B_{1}} s_{B_{2}}\right\rangle \equiv\left|\underline{s_{A_{1}} s_{A_{2}}} 00 \ldots 0 s_{B_{1}} s_{B_{2}}\right\rangle$ with $\left.s_{i} \in\{0,1\}\right)$

$$
\begin{aligned}
& \left|\Psi_{1}(0)\right\rangle=|1100\rangle \equiv \mid \underline{1100 \ldots 0 \underline{00}\rangle}, \\
& \left|\Psi_{2}(0)\right\rangle=|1010\rangle \equiv \mid \underline{1000 \ldots 0 \underline{010}\rangle}, \\
& \left|\Psi_{3}(0)\right\rangle=|1001\rangle \equiv \mid \underline{1000 \ldots 0 \underline{001}\rangle}, \\
& \left|\Psi_{4}(0)\right\rangle=|0110\rangle \equiv \mid \underline{0100 \ldots 0 \underline{010}\rangle} .
\end{aligned}
$$

Note that the remaining options $|0011\rangle$ and $|0101\rangle$ are symmetric to $\left|\Psi_{1}(0)\right\rangle$ and $\left|\Psi_{2}(0)\right\rangle$, respectively. 


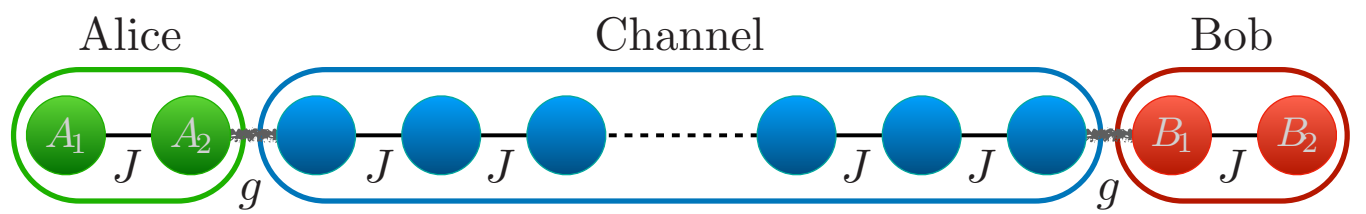

FIG. 1. Alice $(A)$ and Bob $(B)$ each has access to a pair of qubits located at the opposite edges of a quantum channel. Their aim is to generate, via the natural dynamics of the spin chain, an entangled state of the form, e.g., $\left|\Phi_{\theta}^{k_{1}}\right\rangle_{A_{1} B_{2}} \otimes\left|\Phi_{\theta}^{k_{2}}\right\rangle_{A_{2} B_{1}}$, that is, the tensor product of two Bell states, to be used as a resource for two-qubit teleportation.

Equation (1) can be mapped to a spinless fermion model via the Jordan-Wigner transformation [49]

$$
H=\sum_{i=1}^{N-1} J_{i}\left(\hat{c}_{i}^{\dagger} \hat{c}_{i+1}+\hat{c}_{i+1}^{\dagger} \hat{c}_{i}\right),
$$

where $N=M+4$ and $\hat{c}_{i}^{\dagger}\left(\hat{c}_{i}\right)$ is the fermionic creation (annihilation) operator at site $i$. Because of the quadratic nature of the Hamiltonian, the one-particle spectrum is sufficient to describe its full dynamics. Denoting by $\varepsilon_{k}$ and $\left|\varepsilon_{k}\right\rangle=\hat{c}_{k}^{\dagger}|\{0\}\rangle$ the single-particle $k$ th energy eigenvalue and its corresponding eigenvector (with $|\{0\}\rangle$ being the vacuum state), the full Hamiltonian operator acting on a $2^{N}$-dimensional Hilbert space is easily decomposed into a direct sum over all particle number-conserving invariant subspaces $H=\bigoplus_{n=1}^{N} H_{n}$, where

$$
\begin{aligned}
H_{n}= & \sum_{k_{1}<k_{2}<\cdots<k_{n}=1}^{N}\left(\varepsilon_{k_{1}}+\varepsilon_{k_{2}}+\cdots+\varepsilon_{k_{n}}\right) \\
& \times \hat{c}_{k_{1}}^{\dagger} \hat{c}_{k_{2}}^{\dagger} \cdots \hat{c}_{k_{n}}^{\dagger}|\{0\}\rangle\langle\{0\}| \hat{c}_{k_{n}} \cdots \hat{c}_{k_{2}} \hat{c}_{k_{1}} .
\end{aligned}
$$

Each $H_{n}$ can then be constructed quite simply once the singleparticle spectrum is known. Notice that the specific ordering of the $k_{i}$ 's in the sum of Eq. (9) is taken in such a way that unwanted phase factors do not arise when mapping back into spin operators via the inverse Jordan-Wigner transformation. Every invariant subspace is spanned by a set of states having a fixed number of flipped spins. Hence, single-particle states $|j\rangle=\hat{c}_{j}^{\dagger}|\{0\}\rangle$ are obtained by flipping the $j$ th spin of the system, two-particle states $|j i\rangle={\hat{c_{j}}}_{j}^{\dagger} \hat{c}_{i}^{\dagger}|\{0\}\rangle$ are created by flipping the $j$ th and $i$ th spins of the chain (with $j<i$ ), and so forth. The noninteracting nature of the fermionic Hamiltonian in Eq. (9) allows one to reduce the two-particle transition amplitudes $h_{n m}^{p q}(t)=\left\langle p q\left|e^{-i t H_{2}}\right| n m\right\rangle$ to determinants of matrices whose elements are single-particle transition amplitudes $f_{i}^{j}(t)=\left\langle j\left|e^{-i t H_{1}}\right| i\right\rangle$, where $i=\{n, m\}$ and $j=\{p, q\}$ (see, e.g., Ref. $[42,43])$,

$$
h_{n m}^{p q}(t)=\left|\begin{array}{ll}
f_{n}^{p}(t) & f_{n}^{q}(t) \\
f_{m}^{p}(t) & f_{m}^{q}(t)
\end{array}\right| .
$$

Consequently, the evolved state in the two-particle sector results in

$$
|\Psi(t)\rangle=\sum_{p<q=1}^{N} h_{n_{0} m_{0}}^{p q}(t)|p q\rangle,
$$

for an initial state $\left|n_{0} m_{0}\right\rangle$.

Notwithstanding we are able to solve the exact full dynamics of Hamiltonian (1) numerically, it is instructive to rely on a perturbative approach due to the presence of weak couplings $g$ in order to derive an effective Hamiltonian. This allows us to identify more easily the peculiar dynamical behavior behind the generation of highly entangled states between blocks $A$ and $B$.

\section{PERTURbative ANALYSIS}

Similarly to the one-particle subspace dynamics, the model supports one- and two-particle Rabi-like oscillations between its edge spins. This has been used to generate a Bell state in the one-particle subspace in an $X X$-type spin- $\frac{1}{2}$ chain with an even number of sites and a single weakly coupled spin residing at each end, given one gets $|10\rangle \rightarrow \frac{1}{\sqrt{2}}(|10\rangle-|01\rangle)$ shared between them at half the QST time [8].

However, a straightforward extension involving two noninteracting edge spins, such as proposed in Ref. [47], each weakly coupled to the quantum channel, does not yield a tensor product of Bell states. Indeed, starting from, e.g., $|1100\rangle$, because of the permutation symmetry of the edge spins, the amplitudes of states $\{|1001\rangle,|1010\rangle,|0101\rangle,|0110\rangle\}$ have to be equal at all times, thereby preventing the generation of any Bell state between both end blocks.

Turning our attention back to the system arrangement displayed in Fig. 1, where the edge spins are interacting, in the $n=2$ flipped-spin subspace, the states whose dynamics we are about to investigate are listed in Eqs. (4)-(7). Given the dynamics is restricted to the two-excitation subspace and that $g \ll J$, our task now is to carry out a perturbative approach in order to derive an effective Hamiltonian involving only the six possible configurations spanning over both edge blocks ( $A$ and $B)$, that is, $\left\{\left|A_{1} B_{1}\right\rangle,\left|A_{1} B_{2}\right\rangle,\left|A_{2} B_{1}\right\rangle,\left|A_{2} B_{2}\right\rangle,\left|A_{1} A_{2}\right\rangle,\left|B_{1} B_{2}\right\rangle\right\}$ using the occupation-site index notation $|j i\rangle=\hat{c}_{j}^{\dagger} \hat{c}_{i}^{\dagger}|\{0\}\rangle$.

\section{A. Effective description}

Let us split Hamiltonian (8) into $H=H_{0}+H_{\mathrm{ch}}+H_{I}$, where

$$
\begin{gathered}
H_{0}=J\left(c_{A_{1}}^{\dagger} c_{A_{2}}+c_{B_{1}}^{\dagger} c_{B_{2}}+\text { H.c. }\right), \\
H_{\mathrm{ch}}=\sum_{i=1}^{M-1} J\left(c_{i}^{\dagger} c_{i+1}+\text { H.c. }\right), \\
H_{I}=g\left(c_{A_{2}}^{\dagger} c_{1}+c_{M}^{\dagger} c_{B_{1}}+\text { H.c. }\right)
\end{gathered}
$$


The effective Hamiltonian can be obtained via a second-order perturbation method that gives [50]

$$
\begin{aligned}
\left\langle\psi_{0, i}\left|H_{\mathrm{eff}}\right| \psi_{0, j}\right\rangle= & E_{j} \delta_{i, j}-\frac{1}{2} \sum_{k} \\
& \times\left[\frac{\left(H_{I}\right)_{i k}\left(H_{I}\right)_{k j}}{\lambda_{k}-E_{i}}+\frac{\left(H_{I}\right)_{i k}\left(H_{I}\right)_{k j}}{\lambda_{k}-E_{j}}\right],
\end{aligned}
$$

where $\left(H_{I}\right)_{i k} \equiv\left\langle\psi_{0, i}\left|H_{I}\right| \lambda_{k}\right\rangle$, with $\left\{\left|\psi_{0, i}\right\rangle\right\}$ and $\left\{\left|\lambda_{k}\right\rangle\right\}$ being, respectively, the eigenstates of $H_{0}$ and $H_{\mathrm{ch}}$ with corresponding energies $\left\{E_{i}\right\}$ and $\left\{\lambda_{k}\right\}$.

The unperturbed eigenstates of the subsystem of interest, that is, blocks $A$ and $B$, read

$$
\begin{aligned}
& \left|\psi_{0,1}\right\rangle=\left|A_{+} B_{+}\right\rangle, \\
& \left|\psi_{0,2}\right\rangle=\left|A_{+} B_{-}\right\rangle, \\
& \left|\psi_{0,3}\right\rangle=\left|A_{-} B_{+}\right\rangle, \\
& \left|\psi_{0,4}\right\rangle=\left|A_{-} B_{-}\right\rangle, \\
& \left|\psi_{0,5}\right\rangle=\left|A_{1} A_{2}\right\rangle, \\
& \left|\psi_{0,6}\right\rangle=\left|B_{1} B_{2}\right\rangle,
\end{aligned}
$$

where $\left|A_{\mu} B_{v}\right\rangle=\left(\left|A_{1}\right\rangle+\mu\left|A_{2}\right\rangle\right) \otimes\left(\left|B_{1}\right\rangle+\nu\left|B_{2}\right\rangle\right) / 2$, with $\mu, \nu= \pm 1$. Their corresponding eigenvalues are $E_{1}=2 J$, $E_{4}=-2 J$, and $E_{2,3,5,6}=0$.

The single-particle eigenstates of the channel Hamiltonian $H_{\text {ch }}$ are

$$
\left|\epsilon_{m}\right\rangle=\sqrt{\frac{2}{M+1}} \sum_{x=1}^{M} \sin \left(\frac{\pi m x}{M+1}\right)|x\rangle,
$$

with energies $\epsilon_{m}=2 J \cos \left(\frac{\pi m}{M+1}\right)$. Then one can construct $4 \times$ $M$ unperturbed states as $\left|\lambda_{k=(l, m)}\right\rangle=\left|\eta_{l}\right\rangle\left|\epsilon_{m}\right\rangle(l=1,2,3,4$ and $m=1, \ldots, M)$, with $\left|\eta_{1,2}\right\rangle=\left(\left|A_{1}\right\rangle \pm\left|A_{2}\right\rangle\right) / \sqrt{2}$ and $\left|\eta_{3,4}\right\rangle=\left(\left|B_{1}\right\rangle \pm\left|B_{2}\right\rangle\right) / \sqrt{2}$. The corresponding eigenvalues read $\lambda_{l, m}=\epsilon_{m}+J$ for $l=1,3$ and $\lambda_{l, m}=\epsilon_{m}-J$ for $l=$ 2,4 . The remaining unperturbed eigenstates involve linear combinations of states containing no excitations in either block $A$ or $B$. Those provide no contribution to the sum in Eq. (15) given $\left\langle\psi_{0, i}\left|H_{I}\right| x_{1} x_{2}\right\rangle=0$ for all $i$ and $x_{1}, x_{2} \in$ $\{1, \ldots, M\}$.

With all the above relations at hand, one is able to evaluate the matrix elements of $H_{\text {eff }}$ through Eq. (15). When doing so, it is possible to show that all of them are functions of four parameters, namely,

$$
\Lambda_{1}^{ \pm} \equiv \frac{g^{2}}{2} \sum_{m} \frac{a_{m}^{2}}{\epsilon_{m} \pm J}, \quad \Lambda_{2}^{ \pm} \equiv \frac{g^{2}}{2} \sum_{m} \frac{a_{m}^{2} e^{2 i \theta_{m}}}{\epsilon_{m} \pm J},
$$

for a mirror-symmetric channel fulfilling $\left|\alpha_{1}^{m}\right|=\left|\alpha_{M}^{m}\right|=a_{m}$ and $\left(\alpha_{1}^{m}\right)=\left(\alpha_{M}^{m}\right)^{*}=a_{m} e^{i \theta_{m}}$, with $\alpha_{x}^{m} \equiv\left\langle x \mid \epsilon_{m}\right\rangle$ [51]. The expressions above yield $\Lambda_{1}^{ \pm}=0$ and $\Lambda_{2}^{ \pm}=-g^{2} / 2 J$ if $M=$ $6 n(n=1,2, \ldots)$ and $\Lambda_{1}^{ \pm}= \pm g^{2} / 2 J$ and $\Lambda_{2}^{ \pm}=g^{2} / 2 J$ for $M=6 n+4$. We also point out that the above perturbation approach is not valid for $M=6 n+2$ given it yields $\epsilon_{m}= \pm J$, thereby causing divergence of the sums above. Without loss of

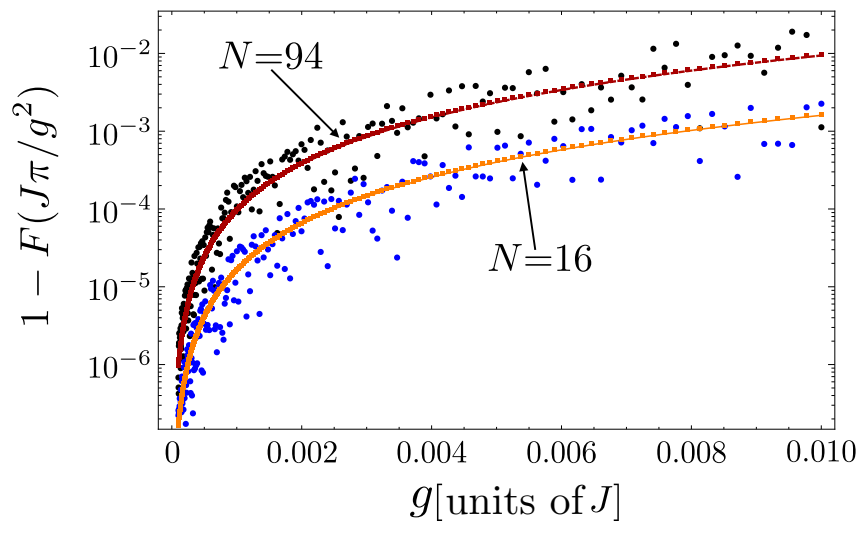

FIG. 2. Infidelity between exact dynamics and the perturbative one vs the weak coupling $g$ (in units of $J$ ) for $N=94$ and $N=16$ evaluated at $t=\pi J / g^{2}$. We take as the initial state $\left|\Psi_{1}(0)\right\rangle$.

generality, though, we consider $M=6 n$ for the remainder of this paper.

After working out every term of the effective Hamiltonian via Eq. (15), its matrix form written in basis $\left\{\left|\psi_{0, i}\right\rangle\right\}$ reads

$$
H_{\mathrm{eff}}=\left(\begin{array}{cccccc}
2 J & 0 & 0 & 0 & \frac{g^{2}}{2 J} & \frac{g^{2}}{2 J} \\
0 & 0 & 0 & 0 & \frac{g^{2}}{2 J} & -\frac{g^{2}}{2 J} \\
0 & 0 & 0 & 0 & \frac{g^{2}}{2 J} & -\frac{g^{2}}{2 J} \\
0 & 0 & 0 & -2 J & \frac{g^{2}}{2 J} & \frac{g^{2}}{2 J} \\
\frac{g^{2}}{2 J} & \frac{g^{2}}{2 J} & \frac{g^{2}}{2 J} & \frac{g^{2}}{2 J} & 0 & 0 \\
\frac{g^{2}}{2 J} & -\frac{g^{2}}{2 J} & -\frac{g^{2}}{2 J} & \frac{g^{2}}{2 J} & 0 & 0
\end{array}\right) .
$$

We finally express its eigenvectors and corresponding eigenvalues in terms of $\left\{\left|s_{A_{1}} s_{A_{2}} s_{B_{1}} s_{B_{2}}\right\rangle\right\}$, recalling that, e.g., $\left|A_{1} B_{1}\right\rangle \equiv|1010\rangle$,

$$
\begin{gathered}
\left|\xi_{1}\right\rangle \approx \frac{1}{2}(|1010\rangle+|1001\rangle+|0110\rangle+|0101\rangle), \\
\left|\xi_{2}\right\rangle \approx \frac{1}{2}(|1010\rangle-|1001\rangle-|0110\rangle+|0101\rangle), \\
\left|\xi_{3}\right\rangle \approx \frac{1}{\sqrt{2}}(|1100\rangle+|0011\rangle), \\
\left|\xi_{4}\right\rangle=\frac{1}{\sqrt{2}}(-|1001\rangle+|0110\rangle), \\
\left|\xi_{5}\right\rangle=\frac{1}{2}(|1010\rangle-|0101\rangle+|1100\rangle-|0011\rangle), \\
\left|\xi_{6}\right\rangle=\frac{1}{2}(|1010\rangle-|0101\rangle-|1100\rangle+|0011\rangle),
\end{gathered}
$$

with $\xi_{1} \approx 2 J, \xi_{2} \approx-2 J, \xi_{3}=\xi_{4}=0, \xi_{5}=g^{2} / J$, and $\xi_{6}=$ $-g^{2} / J$. We stick to the above notation hereafter.

In Fig. 2 we report the infidelity between the states obtained via Eqs. (8) and (24) taken at a specific time for the initial state $\left|\Psi_{1}(0)\right\rangle$ and different values of $g$ and $N$. We see that the infidelity scales as $N g^{2}$, thus validating the second-order perturbation approach for $g \ll \frac{1}{\sqrt{N}}$. As we are interested in a protocol generating long-distance entanglement between the qubits in the sender block and those in the receiver block, we have reported the infidelity between the exact and perturbative dynamics only at the time $t=\frac{\pi J}{g}$, corresponding to the first half of the Rabi-like oscillation of the excitations. 


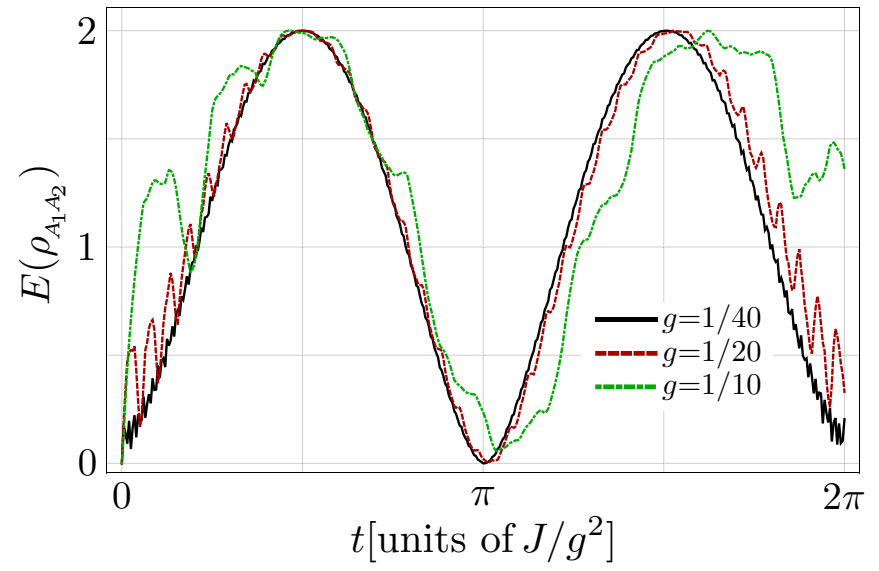

FIG. 3. Exact time evolution of the entanglement entropy $E\left(\rho_{A_{1} A_{2}}\right)$ for $N=22,\left|\Psi_{1}(0)\right\rangle=|1100\rangle$, and different values of $g$. Maximum entanglement is achieved at times $t^{*}=\frac{(2 n+1) \pi J}{2 g^{2}}$, with $n$ being a positive integer, when the state can be expressed as a tensor product of Bell states shared by pairs $\left(A_{1}, B_{2}\right)$ and $\left(A_{2}, B_{1}\right)$.

\section{B. Generation of Bell product states}

We are now ready to track down the time evolution of the initial states displayed in Eqs. (4)-(7) in light of the second-order perturbation theory and check whether a tensor product of Bell states can be achieved involving blocks $A$ and $B$. We stress that the effective description in Eq. (24) entails no excitation within the channel at any time.

According to the eigenstates obtained above, we arrive at the following dynamics for $\left|\Psi_{1}(0)\right\rangle=|1100\rangle$,

$$
\begin{aligned}
\left|\Psi_{1}(t)\right\rangle= & \frac{1}{2}\left[\left(1-\cos \frac{g^{2} t}{J}\right)|0011\rangle+i \sin \frac{g^{2} t}{J}|0101\rangle\right. \\
& \left.-i \sin \frac{g^{2} t}{J}|1010\rangle+\left(1+\cos \frac{g^{2} t}{J}\right)|1100\rangle\right] .
\end{aligned}
$$

Given the above is a pure state, we can evaluate the amount of entanglement block $A$ is sharing with block $B$ by means of the entanglement entropy $E\left(\rho_{A_{1} A_{2}}\right)=\operatorname{Tr}\left[\rho_{A_{1} A_{2}} \log _{2} \rho_{A_{1} A_{2}}\right]$, with $\rho_{A_{1} A_{2}}(t)=\operatorname{Tr}_{B_{1} B_{2}}\left(\left|\Psi_{1}(t)\right\rangle\left\langle\Psi_{1}(t)\right|\right)$, which is reported in Fig. 3, wherein we check via exact diagonalization of the full Hamiltonian, Eq. (1), that it reaches the maximum value attainable for two qubits, $E=2$, at $t^{*}=\frac{(2 n+1) \pi J}{2 g^{2}}$, with $n=$ $0,1,2, \ldots$. Notice that after many Rabi-like oscillations $n$, the perturbative dynamics no longer faithfully reproduces the exact dynamics. This is due to the fact that leakage of the excitations in the chain is building up with time and, in the longtime regime, Rabi-like oscillations eventually are completely suppressed. Nevertheless, in any practical implementation of our protocol, the generated entanglement would be exploited after the first few oscillations, where the agreement between exact and perturbative dynamics is excellent. At such times, the state of Eq. (31) reads

$$
\begin{aligned}
\left|\Psi_{1}\left(t^{*}\right)\right\rangle= & \frac{1}{2}\left(|0011\rangle+(-1)^{n} i|0101\rangle\right. \\
& \left.+(-1)^{n+1} i|1010\rangle+|1100\rangle\right) .
\end{aligned}
$$

This state can be also written as a tensor product of two Bell states between pairs $\left(A_{1}, B_{2}\right)$ and $\left(A_{2}, B_{1}\right)$, namely $\left|\Psi_{1}\left(t^{*}\right)\right\rangle=$ $\left|\Phi_{\theta_{n}}^{1}\right\rangle_{A_{1} B_{2}} \otimes\left|\Phi_{\theta_{n}}^{2}\right\rangle_{A_{2} B_{1}}$, where

$$
\begin{aligned}
\left|\Phi_{\theta_{n}}^{1}\right\rangle_{A_{1} B_{2}} & =\frac{1}{\sqrt{2}}\left(|01\rangle+(-1)^{n+1} i|10\rangle\right), \\
\left|\Phi_{\theta_{n}}^{2}\right\rangle_{A_{2} B_{1}} & =\frac{1}{\sqrt{2}}\left(|01\rangle+(-1)^{n} i|10\rangle\right),
\end{aligned}
$$

and $\theta_{n}=-\pi \frac{2 n+1}{2}$.

Although the state in Eq. (32) is a legitimate one for two-qubit teleportation, Alice may apply a single-qubit phase gate $R\left(\frac{\pi}{2}\right)$ to retrieve the standard Bell states and subsequently follow the protocol addressed in Ref. [28] to carry out the teleportation. Otherwise, as pointed out in Ref. [12], there will be a different set of two local unitary operations Bob has to perform on each of his qubits which we report in the following section.

We reach to a similar scenario starting from $\left|\Psi_{2}(0)\right\rangle=$ $|1010\rangle$,

$$
\begin{aligned}
\left|\Psi_{2}(t)\right\rangle= & \frac{1}{2}\left[\left(\cos 2 J t+\cos \frac{g^{2} t}{J}\right)|1010\rangle\right. \\
& +\left(\cos 2 J t-\cos \frac{g^{2} t}{J}\right)|0101\rangle \\
& -i \sin 2 J t(|1001\rangle+|0110\rangle) \\
& \left.-i \sin \frac{g^{2} t}{J}(|1100\rangle-|0011\rangle)\right],
\end{aligned}
$$

with maximum entanglement entropy $E\left(\rho_{A_{1} A_{2}}\right)$ at the same time $t^{*}=\frac{(2 n+1) \pi J}{2 g^{2}}$, when the state reads

$$
\begin{aligned}
\left|\Psi_{2}\left(t^{*}\right)\right\rangle= & \frac{1}{2}\left[i(-1)^{n}(|0011\rangle-|1100\rangle)\right. \\
& +\cos 2 J t^{*}(|0101\rangle+|1010\rangle) \\
& \left.-i \sin 2 J t^{*}(|0110\rangle+|1001\rangle)\right] .
\end{aligned}
$$

If we now assume that the ratio $J^{2} / g^{2}$ is commensurate such that $2 J t^{*}=2 m \pi$ or $2 J t^{*}=(2 m+1) \pi$, we have that the cosine and sine functions yield, respectively, \pm 1 and 0 . The state in Eq. (36) thus becomes

$$
\begin{aligned}
\left|\Psi_{2}\left(t^{*}\right)\right\rangle= & \frac{1}{2}\left[i(-1)^{n}(|0011\rangle|1100\rangle)\right. \\
& \left.+\mu_{n}(|0101\rangle+|1010\rangle)\right],
\end{aligned}
$$

with $\mu_{n} \equiv \operatorname{sgn}\left[\cos 2 J t^{*}\right]$, which can be readily seen to be the product state $\left|\Psi_{2}\left(t^{*}\right)\right\rangle=i(-1)^{n}\left|\Phi_{\eta}^{1}\right\rangle_{A_{1} B_{2}} \otimes\left|\Phi_{\eta}^{1}\right\rangle_{A_{2} B_{1}}$, where

$$
\left|\Phi_{\eta}^{1}\right\rangle_{A_{1} B_{2}}=\frac{1}{\sqrt{2}}\left(|01\rangle-\mu_{n}(-1)^{n} i|10\rangle\right),
$$

and $\eta=\pi \frac{\mu_{n}}{2}$. Similarly, for $2 J t^{*}=\pi / 2+2 n \pi$ or $2 J t^{*}=$ $3 \pi / 2+2 n \pi$, cosine and sine functions give, respectively, 0 and \pm 1 , and the state in $\mathrm{Eq}$ (36) evolves into $\left|\Psi_{2}\left(t^{*}\right)\right\rangle=$ $i(-1)^{n}\left|\Phi_{\eta}^{1}\right\rangle_{A_{1} B_{1}} \otimes\left|\Phi_{\eta}^{1}\right\rangle_{A_{2} B_{2}}$. For times different from those reported above, although the entanglement entropy is maximum, the state cannot be decomposed into a tensor product of Bell states.

The two remaining initial states in our investigation, $\left|\Psi_{3}(0)\right\rangle=|1001\rangle$ and $\left|\Psi_{4}(0)\right\rangle=|0110\rangle$, do not yield any entanglement between block $A$ and $B$ at any time, that is, 
$E\left[\rho_{A_{1} A_{2}}(t)\right]=0$. It is interesting to note that their dynamics does not even involve $g$ in the second-order perturbation expansion.

\section{ENTANGLEMENT OF TELEPORTATION}

The one-qubit teleportation protocol establishes that Alice and Bob share a pair of qubits in a maximally entangled (Bell) state $\left|\Phi_{\theta}^{k}\right\rangle_{A B}$ [cf. Eqs. (2) and (3)] and that the former performs a Bell measurement on her shared qubit and an unknown one $|\varphi\rangle_{X}$. It is thus convenient to express the initial state of the protocol as

$$
|\varphi\rangle_{X} \otimes\left|\Phi_{\theta}^{k}\right\rangle_{A B}=\frac{1}{2} \sum_{j=1}^{4}\left|\Phi_{\theta}^{j}\right\rangle_{X A} \otimes \mathcal{O}_{j}^{k}|\varphi\rangle_{B},
$$

where the operators $\mathcal{O}_{j}^{k}$ depend on the initially shared entangled state. Alice must now perform a Bell measurement depending on the relative phase $\theta$. The state reduces, with equal probability, to one of the Bell states and Alice classically communicates her result to Bob. Depending on the outcome, he is able to recover the unknown state $|\varphi\rangle_{B}$ performing the right operation $\tilde{\mathcal{O}}_{j}^{k}$ (according to initial shared state) among the set

$$
\begin{gathered}
\tilde{\mathcal{O}}_{j}^{1}=\left\{-R(\theta), R(\theta) \sigma^{z}, \sigma^{x}, \sigma^{z} \sigma^{x}\right\}, \\
\tilde{\mathcal{O}}_{j}^{2}=\left\{-R(\theta) \sigma^{z}, R(\theta), \sigma^{z} \sigma^{x}, \sigma^{x}\right\}, \\
\tilde{\mathcal{O}}_{j}^{3}=\left\{-R(-\theta) \sigma^{x},-R(-\theta) \sigma^{z} \sigma^{x}, \mathbf{I},-\sigma^{z}\right\}, \\
\tilde{\mathcal{O}}_{j}^{4}=\left\{R(-\theta) \sigma^{z} \sigma^{x}, R(-\theta) \sigma^{x},-\sigma^{z}, \mathbf{I}\right\},
\end{gathered}
$$

with

$$
R(\theta)=\left(\begin{array}{cc}
e^{i \theta} & 0 \\
0 & e^{-i \theta}
\end{array}\right) .
$$

Observe that $\tilde{\mathcal{O}}_{j}^{k}$ is simply the inverse of $\mathcal{O}_{j}^{k}$ in Eq. (39). The quantum resource for this protocol is a maximally entangled state, i.e., the Bell state of two qubits.

For the two-qubit teleportation protocol let us suppose Alice and Bob share the state $\left|\Phi_{\theta}^{k_{1}}\right\rangle_{A_{1} B_{2}} \otimes\left|\Phi_{\theta}^{k_{2}}\right\rangle_{A_{2} B_{1}}$ and Alice wants to teleport an arbitrary two-qubit state $|\varphi\rangle_{X Y}=a|00\rangle+$ $b|01\rangle+c|10\rangle+d|11\rangle$. The initial state of the protocol can then be written as

$$
\begin{aligned}
|\varphi\rangle_{X Y} & \otimes\left|\Phi_{\theta}^{k_{1}}\right\rangle_{A_{1} B_{2}} \otimes\left|\Phi_{\theta}^{k_{2}}\right\rangle_{A_{2} B_{1}} \\
& =\frac{1}{4} \sum_{j_{1}, j_{2}=1}^{4}\left|\Phi_{\theta}^{j_{1}}\right\rangle_{X A_{1}} \otimes\left|\Phi_{\theta}^{j_{2}}\right\rangle_{Y A_{2}} \otimes\left(\mathcal{O}_{j_{1}}^{k_{2}} \otimes \mathcal{O}_{j_{2}}^{k_{2}}\right)|\varphi\rangle_{B_{1} B_{2}} .
\end{aligned}
$$

As a consequence, a measurement in the generalized Bell basis given above on Alice's pairs of qubits $\left(X, A_{1}\right)$ and $\left(Y, A_{2}\right)$ reduces the scheme to the standard single-qubit teleportation protocol for each qubit $X$ and $Y$.

In Ref. [28], the entanglement of teleportation (EoT) $E_{T}(|\Phi\rangle)$ has been introduced as a measure of the usefulness of a $2 n$-qubit pure state $|\Phi\rangle$ for $n$-qubit teleportation. In the following we report its expression for the case of four qubits. The EoT is based on the generalized concurrence
[52], $C(|\Phi\rangle)=|\langle\Phi \mid \tilde{\Phi}\rangle|$, where $|\tilde{\Phi}\rangle=\hat{\sigma}_{A_{1}}^{y} \hat{\sigma}_{A_{2}}^{y} \hat{\sigma}_{B_{1}}^{y} \hat{\sigma}_{B_{2}}^{y}|\Phi\rangle^{*}$ and the state is expressed in the computational basis. Hence, $E_{T}(|\Phi\rangle)=\frac{1}{16} \sum_{i=1}^{16} C\left(\left|\Phi^{(i)}\right\rangle\right)$, where $\left|\Phi^{(i)}\right\rangle$ are all the orthogonal states that can be obtained from $|\Phi\rangle$ by applying certain single-qubit unitary operations, as reported in Ref. [28]. Let us point out that the EoT is independent of the choice of basis as long as each of the 16 basis states are composed of a tensor product of maximally entangled states. Straightforward calculations show that in the case of $\theta=\frac{\pi}{2}$ the states reported in Eqs. (32) and (37) have unit EoT.

Although the states obtained by the full and effective dynamics have vanishing infidelity, as shown in Fig. 2, let us also compare, for the sake of completeness, the efficiency of the teleportation protocol performed via the exact and the reduced states as, in principle, states with high fidelity may not share the same resources [53]. To this aim we report the fidelity of teleportation $F_{T}$ by means of the full and the effective Hamiltonians reported in the previous sections, in Eqs. (8) and (24), respectively. The fidelity of teleportation is given by the overlap of the unknown state to be teleported, say, $\left|\varphi_{\text {in }}\right\rangle$, and Bob's output state $\hat{\rho}_{\text {out }}$, that is, $F_{T}=\left\langle\varphi_{\text {in }}\left|\hat{\rho}_{\text {out }}\right| \varphi_{\text {in }}\right\rangle$. Using the two-qubit parametrization as in Ref. [42],

$$
\begin{aligned}
|\varphi\rangle_{\text {in }}= & \sqrt{\frac{1-s}{2}}\left(\cos \frac{\theta_{1}}{2}|0\rangle+e^{i \phi_{1}} \sin \frac{\theta_{1}}{2}|1\rangle\right) \\
& \otimes\left(\cos \frac{\theta_{2}}{2}|0\rangle+e^{i \phi_{2}} \sin \frac{\theta_{2}}{2}|1\rangle\right) \\
& +\sqrt{\frac{1+s}{2}}\left(e^{-i \phi_{1}} \sin \frac{\theta_{1}}{2}|0\rangle-\cos \frac{\theta_{1}}{2}|1\rangle\right) \\
& \otimes\left(e^{-i \phi_{2}} \sin \frac{\theta_{2}}{2}|0\rangle-\cos \frac{\theta_{2}}{2}|1\rangle\right),
\end{aligned}
$$

with $0 \leqslant \theta_{1,2} \leqslant \pi, 0 \leqslant \phi_{1,2} \leqslant 2 \pi$, and $-1 \leqslant s \leqslant 1$.

After working out the fidelity of teleportation of such a state, according to the effective Hamiltonian description, we integrate it over all possible inputs to obtain the average fidelity of teleportation,

$$
\bar{F}_{\text {eff }}(t)=\frac{1}{2}-\frac{7}{54} \cos \frac{2 g^{2} t}{J}+\frac{10}{27} \sin \frac{g^{2} t}{J} .
$$

On the other hand, the average $F_{T}$ for the full dynamics reads

$$
\begin{aligned}
\bar{F}_{T}(t)= & \frac{1}{27}\left(7+3\left|h_{12}\right|^{2}+3\left|h_{1 N-1}\right|^{2}+6\left|h_{1 N}\right|^{2}+3\left|h_{N-1 N}\right|^{2}\right. \\
& +3\left|h_{2 N}\right|^{2}-2 \sum_{n=3}^{N-2}\left(\left|h_{1 n}\right|^{2}+\left|h_{2 n}\right|^{2}+\left|h_{n N-1}\right|^{2}+\left|h_{n N}\right|^{2}\right) \\
& +14 \operatorname{Re}\left(h_{12} h_{N-1 N}^{*}-h_{2 N} h_{1 N-1}^{*}\right)+10 \operatorname{Im}\left(h_{12} h_{1 N-1}^{*}\right. \\
& \left.+h_{2 N} h_{N-1 N}^{*}-h_{1 N-1} h_{N-1 N}^{*}-h_{12} h_{2 N}^{*}\right) \\
& \left.-4 \sum_{n=3}^{N-2} \operatorname{Im}\left(h_{1 n} h_{n N}^{*}-h_{2 n} h_{n N-1}^{*}\right)\right)
\end{aligned}
$$

where, for conciseness, $h_{p q}$ stands for $h_{12}^{p q}$ as defined in Eq. (10), and Re (Im) denote the real (imaginary) part. In 


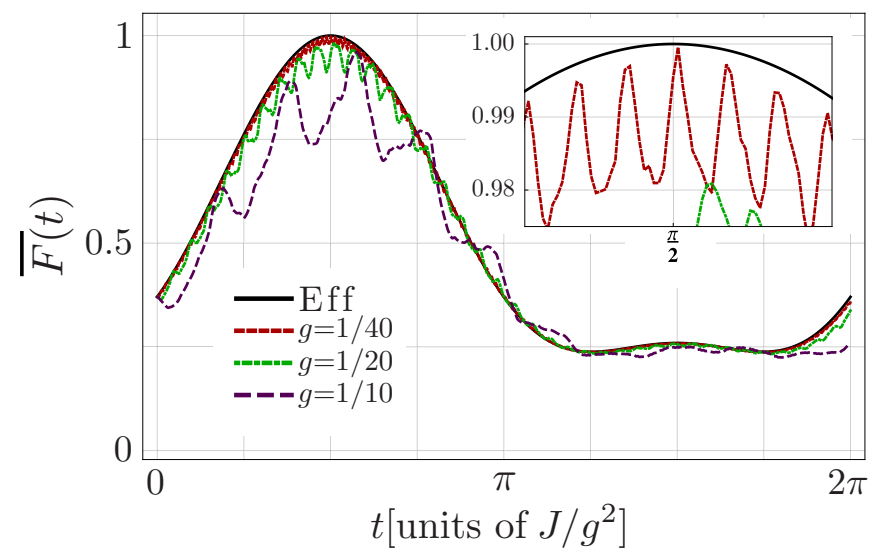

FIG. 4. Average fidelity of teleportation for the full model for a random two-qubit state for various values of $g$. Time is being expressed in units of $J / g^{2}$. The black curve represents the same quantity for the effective dynamics obtained from the second-order perturbation approach.

Fig. 4 we report $\bar{F}_{T}$ for a chain with $N=22$ sites for different values of $g$, comparing it with the effective description $\bar{F}_{\text {eff }}$. It can be seen that already for values of $g / J=1 / 40$, the effective description is faithful and the average fidelity of teleportation is very close to the unit at time $t^{*}$ obtained from the perturbative analysis (see the inset of Fig. 4). From Figs. 3 and 4 we can also infer that, for $g \ll 1$, being the entanglement entropy and the average fidelity of teleportation obtained by the perturbative approach an excellent approximation of the same quantities derived via the exact dynamics, the infidelity of the two respective maps should be negligible at any time, and not only at the times shown in Fig. 2.

Finally, as the resource for two-qubit teleportation may be used at an arbitrary time after $t^{*}$, we consider the case where Alice and Bob decouple, at $t=t^{*}$, their respective blocks from the channel, i.e., set $g=0$. The qubits in each block are still interacting according to $H_{0}$ in Eq. (12), but the entanglement between the blocks remains constant. This can be easily shown as the pair of spins in each block are left, at $t=t^{*}$, in a state close to the maximally mixed state one. As $\left[\hat{H}_{i j}, \hat{\rho}_{i j}\right]=0$, for $i, j=A_{1}, A_{2}$ and $B_{1}, B_{2}$, the entanglement of teleportation also stays almost constant, exhibiting oscillations with less than $1 \%$ of the values at $t=\frac{\pi J}{2 g^{2}}$ (see Fig. 3), as we have numerically verified.

\section{CONCLUSIONS}

We worked out a protocol for generating four-qubit generalized Bell states, to be used in the quantum teleportation of an arbitrary two-qubit state, via the natural Hamiltonian dynamics of a $X X$ spin- $\frac{1}{2}$ chain with weakly coupled end blocks. We derived analytically the effective dynamics of the system in the two-excitation manifold up to second-order perturbation theory.

We found that a simple initialization of the sender and receiver blocks, i.e., a two-spin flip on a overall fully polarized spin background of the quantum channel, results in the generation of the appropriate resource (entanglement) upon which the teleportation protocol will rely on. Note that, at variance with entanglement distribution schemes, where entanglement is shared initially between two pairs of qubits (of which only one belongs to the chain) and the quantum channel is used to distribute the initial entanglement, our protocol generates entanglement via the natural dynamics of the chain and no preexisting entanglement is required.

Motivated by the need, in several quantum information processing tasks, to transfer many-qubit states with a minimum amount of resources, we have taken a step in this direction by implementing the two-qubit case in a quantum channel, which also fulfills the one-qubit scheme. Remarkably, the timescale for sharing a tensor product of two Bell states is the same as that required for a single Bell state, hinting towards the possibility that the generation of an arbitrary tensor product of Bell states, via our protocol, is independent of the wanted number of Bell pairs. This seems to be a consequence of the noninteracting nature of the model, where the many-particle dynamics can be evaluated through single-particle transition amplitudes. This will be addressed in a future project.

Our work was inspired by the idea of using preengineered spin chains for transmitting (and generating) states from one point to another with minimal control, which may find applications in intermediate-scale quantum computations as well [54]. Further extensions of this work should generalize the protocol to cover the generation of resources for $n$-qubit teleportation as well as investigate the effects static disorder and other forms of noise, as well as other ways to perturbatively couple the sender and the receiver blocks to the quantum channel, e.g., using strong local magnetic fields $[41,42,55]$. Finally, considering the high level of control achievable in cold atom settings, we believe that our protocol is within experimental reach [56-58].
[1] S. Bose, Contemp. Phys. 48, 13 (2007).

[2] G. Nikolopoulos and I. Jex, Quantum State Transfer and Network Engineering, Quantum Science and Technology (Springer, Berlin, 2013).

[3] T. J. G. Apollaro, S. Lorenzo, and F. Plastina, Int. J. Mod. Phys. B 27, 1345035 (2013).

[4] S. Ritter, C. Nölleke, C. Hahn, A. Reiserer, A. Neuzner, M. Uphoff, M. Mücke, E. Figueroa, J. Bochmann, and G. Rempe, Nature (London) 484, 195 (2012).

[5] P. Kurpiers, P. Magnard, T. Walter, B. Royer, M. Pechal, J. Heinsoo, Y. Salathé, A. Akin, S. Storz, J.-C. Besse,
S. Gasparinetti, A. Blais, and A. Wallraff, Nature (London) 558, 264 (2018).

[6] S. Bose, Phys. Rev. Lett. 91, 207901 (2003).

[7] M. Christandl, N. Datta, A. Ekert, and A. J. Landahl, Phys. Rev. Lett. 92, 187902 (2004).

[8] A. Wójcik, T. Łuczak, P. Kurzyński, A. Grudka, T. Gdala, and M. Bednarska, Phys. Rev. A 72, 034303 (2005).

[9] G. M. A. Almeida, Phys. Rev. A 98, 012334 (2018).

[10] G. M. A. Almeida, F. A. B. F. de Moura, T. J. G. Apollaro, and M. L. Lyra, Phys. Rev. A 96, 032315 (2017).

[11] A. Kay, Quantum 1, 24 (2017). 
[12] C. H. Bennett, G. Brassard, C. Crépeau, R. Jozsa, A. Peres, and W. K. Wootters, Phys. Rev. Lett. 70, 1895 (1993).

[13] D. Boschi, S. Branca, F. De Martini, L. Hardy, and S. Popescu, Phys. Rev. Lett. 80, 1121 (1998).

[14] H. Bechmann-Pasquinucci and A. Peres, Phys. Rev. Lett. 85, 3313 (2000).

[15] N. J. Cerf, M. Bourennane, A. Karlsson, and N. Gisin, Phys. Rev. Lett. 88, 127902 (2002).

[16] D. Bruß and C. Macchiavello, Phys. Rev. Lett. 88, 127901 (2002).

[17] A. Acin, N. Gisin, and V. Scarani, Quantum Inf. Comput. 3, 563 (2003).

[18] V. Karimipour, A. Bahraminasab, and S. Bagherinezhad, Phys. Rev. A 65, 052331 (2002).

[19] T. Durt, D. Kaszlikowski, J.-L. Chen, and L. C. Kwek, Phys. Rev. A 69, 032313 (2004).

[20] J. Nunn, L. J. Wright, C. Söller, L. Zhang, I. A. Walmsley, and B. J. Smith, Opt. Express 21, 15959 (2013).

[21] J. Mower, Z. Zhang, P. Desjardins, C. Lee, J. H. Shapiro, and D. Englund, Phys. Rev. A 87, 062322 (2013).

[22] C. Lee, Z. Zhang, G. R. Steinbrecher, H. Zhou, J. Mower, T. Zhong, L. Wang, X. Hu, R. D. Horansky, V. B. Verma, A. E. Lita, R. P. Mirin, F. Marsili, M. D. Shaw, S. W. Nam, G. W. Wornell, F. N. C. Wong, J. H. Shapiro, and D. Englund, Phys. Rev. A 90, 062331 (2014).

[23] T. Zhong, H. Zhou, R. D. Horansky, C. Lee, V. B. Verma, A. E. Lita, A. Restelli, J. C. Bienfang, R. P. Mirin, T. Gerrits, S. W. Nam, F. Marsili, M. D. Shaw, Z. Zhang, L. Wang, D. Englund, G. W. Wornell, J. H. Shapiro, and F. N. C. Wong, New J. Phys. 17, 022002 (2015).

[24] R. Beals, S. Brierley, O. Gray, A. W. Harrow, S. Kutin, N. Linden, D. Shepherd, and M. Stather, Proc. R. Soc. London Ser. A 469, 20120686 (2013).

[25] X. Qiang, X. Zhou, K. Aungskunsiri, H. Cable, and J. L. O’Brien, Quantum Sci. Technol. 2, 045002 (2017).

[26] A. Karlsson and M. Bourennane, Phys. Rev. A 58, 4394 (1998).

[27] P. Agrawal and A. Pati, Phys. Rev. A 74, 062320 (2006).

[28] G. Rigolin, Phys. Rev. A 71, 032303 (2005).

[29] F.-G. Deng, Phys. Rev. A 72, 036301 (2005).

[30] Y. Yeo and W. K. Chua, Phys. Rev. Lett. 96, 060502 (2006).

[31] S. Muralidharan and P. K. Panigrahi, Phys. Rev. A 77, 032321 (2008).

[32] M.-J. Zhao, Z.-G. Li, X. Li-Jost, and S.-M. Fei, J. Phys. A: Math. Theor. 45, 405303 (2012).
[33] P.-X. Chen, S.-Y. Zhu, and G.-C. Guo, Phys. Rev. A 74, 032324 (2006).

[34] L. Banchi, A. Bayat, P. Verrucchi, and S. Bose, Phys. Rev. Lett. 106, 140501 (2011).

[35] G. M. A. Almeida, F. Ciccarello, T. J. G. Apollaro, and A. M. C. Souza, Phys. Rev. A 93, 032310 (2016).

[36] C. Di Franco, M. Paternostro, and M. S. Kim, Phys. Rev. A 77, 020303(R) (2008).

[37] M. P. Estarellas, I. D'Amico, and T. P. Spiller, Phys. Rev. A 95, 042335 (2017).

[38] X. Chen, R. Mereau, and D. L. Feder, Phys. Rev. A 93, 012343 (2016).

[39] F. de Pasquale, G. Giorgi, and S. Paganelli, Phys. Rev. Lett. 93, 120502 (2004).

[40] F. de Pasquale, G. L. Giorgi, and S. Paganelli, Phys. Rev. A 71, 042304 (2005).

[41] S. Lorenzo, T. J. G. Apollaro, S. Paganelli, G. M. Palma, and F. Plastina, Phys. Rev. A 91, 042321 (2015).

[42] T. J. G. Apollaro, S. Lorenzo, A. Sindona, S. Paganelli, G. L. Giorgi, and F. Plastina, Phys. Scr. T165, 014036 (2015).

[43] R. Sousa and Y. Omar, New J. Phys. 16, 123003 (2014).

[44] S. Lorenzo, T. J. G. Apollaro, A. Trombettoni, and S. Paganelli, Int. J. Quantum Inf. 15, 1750037 (2017).

[45] P. Lorenz and J. Stolze, Phys. Rev. A 90, 044301 (2014).

[46] H. Verma, L. Chotorlishvili, J. Berakdar, and S. K. Mishra, Europhys. Lett. 119, 30001 (2017).

[47] R. Vieira and G. Rigolin, Phys. Lett. A 382, 2586 (2018).

[48] L. Latmiral, C. Di Franco, P. L. Mennea, and M. S. Kim, Phys. Rev. A 92, 022350 (2015).

[49] E. Lieb, T. Schultz, and D. Mattis, Ann. Phys. 16, 407 (1961).

[50] Y. Li, T. Shi, B. Chen, Z. Song, and C.-P. Sun, Phys. Rev. A 71, 022301 (2005).

[51] L. Banchi and R. Vaia, J. Math. Phys. 54, 043501 (2013).

[52] A. Wong and N. Christensen, Phys. Rev. A 63, 044301 (2001).

[53] M. Bina, A. Mandarino, S. Olivares, and M. G. A. Paris, Phys. Rev. A 89, 012305 (2014).

[54] J. Preskill, Quantum 2, 79 (2018).

[55] S. Lorenzo, T. J. G. Apollaro, A. Sindona, and F. Plastina, Phys. Rev. A 87, 042313 (2013).

[56] A. G. Volosniev, D. Petrosyan, M. Valiente, D. V. Fedorov, A. S. Jensen, and N. T. Zinner, Phys. Rev. A 91, 023620 (2015).

[57] A. A. Khajetoorians, J. Wiebe, B. Chilian, and R. Wiesendanger, Science 332, 1062 (2011).

[58] L. Banchi, J. Fernández-Rossier, C. F. Hirjibehedin, and S. Bose, Phys. Rev. Lett. 118, 147203 (2017). 